\title{
Upbeat nystagmus in a patient with a small medullary infarct
}

\author{
N A R Munro, B Gaymard, S Rivaud, A Majdalani, Ch Pierrot-Deseilligny
}

\begin{abstract}
A 43 year old man presented with decreased sensitivity in the left side of the face and both upper limbs, and with upbeat nystagmus and skew deviation. MRI demonstrated a well defined lesion compatible with an infarct in the left side of the medulla, caudal and ventral to the vestibular nuclei, possibly involving the most caudal of the perihypoglossal nuclei, the nucleus intercalatus. Ocular motor studies, using an infrared system, showed that the nystagmus slow phase decayed exponentially, suggesting a failure of integration for vertical eye movements. Vertical integration might, therefore, be performed partly in the nucleus intercalatus.
\end{abstract}

(尹 Neurol Neurosurg Psychiatry 1993;56:1126-1128)

Upbeat nystagmus is an uncommon clinical finding usually associated with lesions affecting the pontomesencephalic junction, ${ }^{1}$ or the rostral medulla or caudal pons. ${ }^{1-6}$ Less commonly, it has been associated with cerebellar or midbrain lesions, where its association with internuclear ophthalmoplegia is well recognised. ${ }^{78}$ We report a case with upbeat nystagmus and a skew deviation related to a small medullary infarct.

\section{Case report}

A 43 year old man suffered a mild headache and a strange sensation in the left side of his face and in his left hand. Subsequently, he vomited, though there was no vertigo. He discovered that he was veering to the left. On arrival at the hospital, he complained of persistent hiccoughs and double vision. Examination showed an upbeat nystagmus in both eyes, barely visible in the primary position, but more marked on upward gaze. A mild horizontal gaze nystagmus was also present. He had an intermittent skew deviation with hypotropia of the left eye, without evidence of a torsional component. There was a slight increase of the right sided reflexes. Sensitivity to light touch in the left side of the face and in the left hand was decreased, as was sensitivity to pain and temperature on the right side of the body, sparing the face.
Romberg's test showed a tendency to fall to the left. There were no cerebellar signs.

\section{MRI}

A small left sided medullary infarct extending $4 \mathrm{~mm}$ in its rostrocaudal length was demonstrated $5 \mathrm{~mm}$ caudal to the pontomedullary junction (fig 1A,B). The infarct was ovoid, sparing both ventral and dorsal medulla but probably involved the central nuclei of the medulla oblongata, the medullary part of the fifth nerve nucleus, the inferior tip of the nucleus ambiguus, and the spinothalamic tract. Ventrally, the caudal tip of the inferior olive and the pyramids were spared. Dorsally, the medial part of the cuneate nucleus appeared to be affected, while its lateral part and the gracile nucleus were spared. Medially, the lesion extended to the nucleus and tractus solitarius and the nucleus intercalatus and was close to the 12 th nerve nucleus (fig 1C). The rostral tip of this lesion was caudal to the caudal tip of both the vestibular nuclei and the nucleus prepositus hypoglossi.

\section{Eye movement recordings}

Recordings were made 5 days after the initial symptoms. The patient was seated, with head fixed, $80 \mathrm{~cm}$ in front of a semicircular array of closely spaced light emitting diodes. Recordings were made from the right eye using infrared oculography (Skalar "IRIS" system) and were sampled at $250 \mathrm{~Hz}$.

There was an upbeat nystagmus in the primary position which was increased on looking upwards (fig 2), persisting even with prolonged eccentricity of gaze. The character of the slow phase was clearly exponentially decaying. There was a horizontal gaze nystagmus on looking to the right which was just present in the primary position. There was no rebound nystagmus. Vertical smooth pursuit of a target moving sinusoidally with amplitude of $10^{\circ}$ and frequencies of 0.25 and $0.5 \mathrm{~Hz}$ were symmetrical. The gain of horizontal smooth pursuit was slightly reduced towards the left. Lateral and vertical saccades were normal.

\section{Discussion}

This patient suffered from a postural imbalance in the absence of symptoms of rotational vertigo, an upbeat nystagmus, with an expo- 
Figure 1 Lateral $(A)$, dorsal (B), and cross sectional (C) Magnetic resonance images of the pons and medulla. Left sided structures are demonstrated on the left. 1 vestibular nuclei: (a) superior, (b) lateral, (c) inferior, (d) medial; 2 nucleus prepositus hypoglossi; 3 nucleus of Roller; 4 nucleus intercalatus (hatched area); 5 XII nerve nucleus; 6 nucleus and tractus solitarius; 7 gracilis and cuneate nuclei; 8 nucleus and tractus of $V$ nerve; 9 spinothalamic tract; 10 inferior olive; 11 nucleus ambiguus; 12 pyramids. The lesion is demonstrated by light shading and is shown involving the nucleus intercalatus (hatched area).
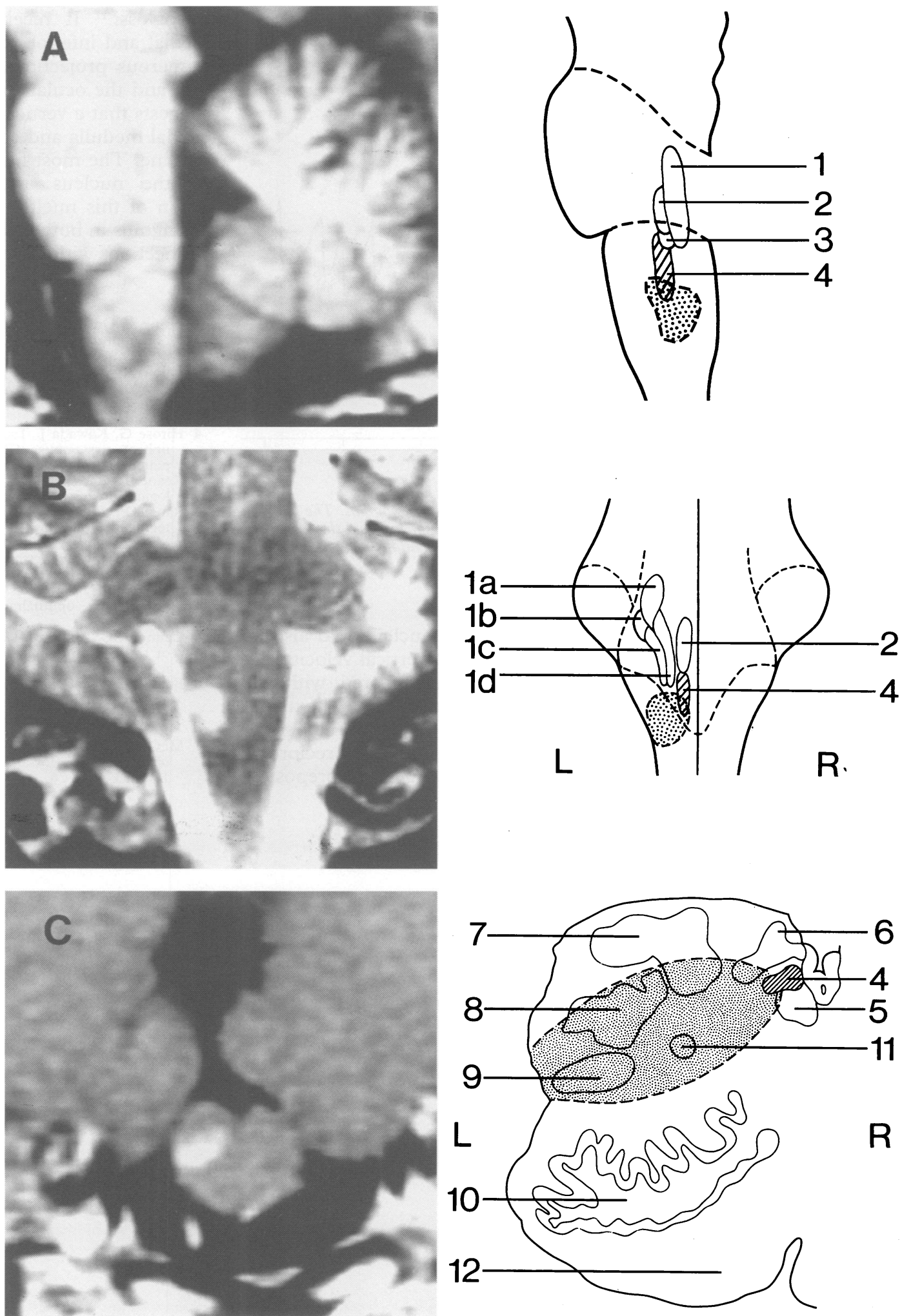

nentially decaying waveform, and an intermittent skew deviation.

Upbeat nystagmus has not so far been reported with a small infarct affecting the caudal dorsolateral medulla. Upbeat nystagmus is not a usual finding in a pure lateral medullary syndrome (Wallenberg's syndrome). However, upbeat nystagmus has been reported in two cases involving the medial medulla and in a patient with a haematoma affecting the central medulla, which had bled several times. ${ }^{25}$ The vestibular system has been implicated in upbeat nystagmus, ${ }^{126}$ but in our patient the lesion was located more ventrally and caudally to the vestibular nuclei. The clear demonstration of exponential decay in the slow phase is strong evidence of impaired integration. ${ }^{9}$ The nucleus prepositus hypoglossi, the largest of the perihypoglossal nuclei, is thought, with the medial vestibular nucleus, to be the integrator for horizontal eye movements. ${ }^{9}$ This nucleus 


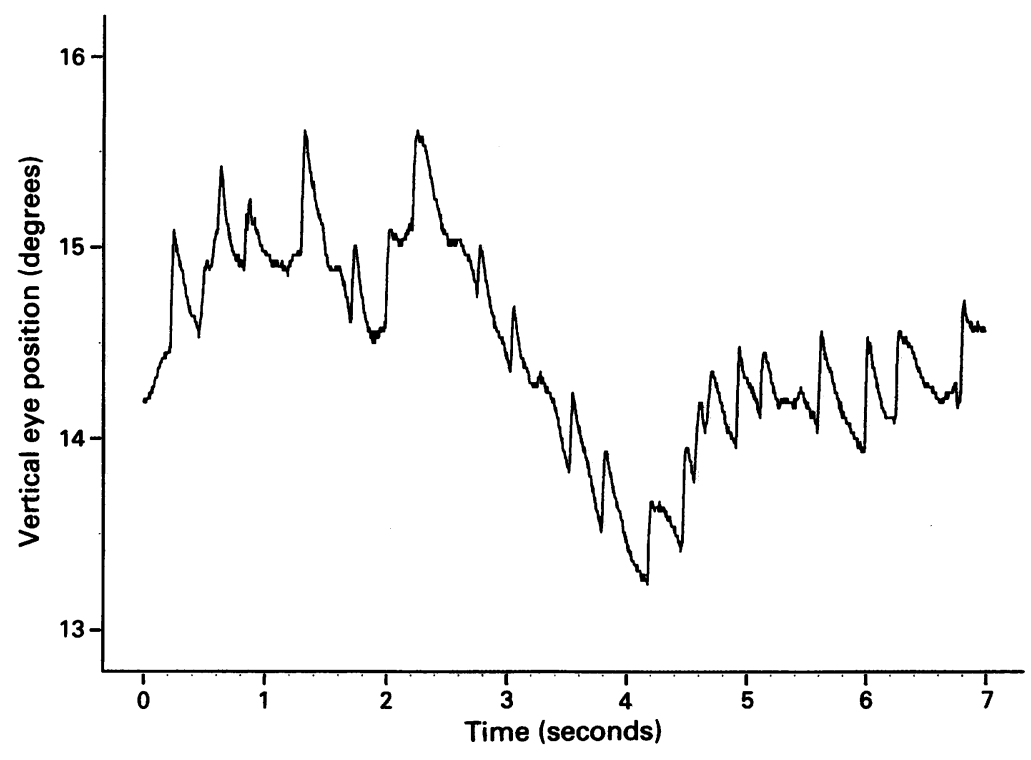

Figure 2 Vertical eye movement recording demonstrating upbeat nystagmus. The patient is fixating a point $15^{\circ}$ above the horizontal. The fast phases are upward and show an amplitude of approximately $0.5^{\circ}$. The slow phases show an exponentially decaying waveform. hypoglossi. ${ }^{11}$ It receives afferents from the medial and inferior vestibular nuclei and has numerous projections including the cerebellum and the ocular motor nuclei. ${ }^{11}$ This case suggests that a vertical integrator exists in the caudal medulla and could be located near the midline. The most likely structure appears to be the nucleus intercalatus. A unilateral lesion of this nucleus could result in upbeat nystagmus in both eyes because of its bilateral connections with other brainstem structures.

1 Fisher A, Gresty M, Chambers B, Rudge P. Primary position upbeating nystagmus. A variety of central positional nystagmus. Brain 1983;106:949-64.

nystagmus. Brain 1983;106:949-64.
Baloh RW, Yee RD. Spontaneous vertical nystagmus. Rev Neurol (Paris) 1989;145:527-32.

3 Daroff RB, Troost BT. Upbeat nystagmus. $f \mathrm{Am} \mathrm{Med}$ Assoc 1973;225:312.

4 Hirose G, Kawada J, Tsukada K, et al. Primary position upbeat nystagmus. Clinicopathologic study of four patients. Acta Otolaryngol (Stockh) 1991;111:357-60.

5 Keane JR, Itabashi HH. Upbeat nystagmus: clinicopathologic study of two patients. Neurology 1987;37:491-4.

6 Ranalli PJ, Sharpe JA. Upbeat nystagmus and the ventral tegmental pathway of the upward vestibulo-ocular reflex. Neurology 1988;38:1329-30.

7 Pawl R. Upbeat nystagmus. F Am Med Assoc 1973; 225-312.

8 Kirkham TH, Katsarkas A An electrographic study of internuclear ophthalmoplegia. Ann Neurol 1977;25: $385-92$

or other perihypoglossal nuclei could also be involved in integration of vertical velocity signals, a function probably shared with the nucleus of $\mathrm{Cajal}^{10}$ in the midbrain. The nucleus intercalatus, the most caudal of the perihypoglossal nuclei, has strong reciprocal connections with the nucleus prepositus 